\title{
Publisher Correction: Reduction of liver fibrosis by rationally designed macromolecular telmisartan prodrugs
}

Matthew R. Golder (D), Jenny Liu, Jannik N. Andersen, Michail V. Shipitsin, Farrukh Vohidov, Hung V.-T. Nguyen, Deborah C. Ehrlich, Sung Jin Huh, Bhavatarini Vangamudi, Kyriakos D. Economides, Allison M. Neenan, James C. Ackley, Joelle Baddour, Sattanathan Paramasivan, Samantha W. Brady, Eric J. Held, Lawrence A. Reiter, Jennifer K. Saucier-Sawyer, Paul W. Kopesky, Donald E. Chickering, Peter Blume-Jensen and Jeremiah A. Johnson (D)

Correction to: Nature Biomedical Engineering https://doi.org/10.1038/s41551-018-0279-x, published online 20 August 2018.

In the version of this Article originally published, the author Peter Blume-Jensen was not denoted as a corresponding author; this has now been amended and the author's email address has been added. The 'Correspondence and requests for materials' statement was similarly affected and has now been updated with the author's initials 'P.B-J.' 